\title{
Animal models of FVIla gene expression: their role in the future development of haemophilia treatment
}

\author{
A. OBERGFELL ${ }^{*}$, T. NICHOLS ${ }^{\dagger}$, and M. EZBAN ${ }^{\ddagger}$ \\ ${ }^{*}$ NovoNordisk, Zurich Oerlikon, Switzerland tDepartment of Pathology, Francis Owen Blood \\ Research Laboratory, University of North Carolina, NC, USA $¥$ NovoNordisk A/S, Bagsværd, \\ Denmark
}

\section{Summary}

The development of inhibitors to replacement factor therapy is a serious complication in the treatment of patients with haemophilia and requires use of bypassing agents to prevent uncontrolled bleeding. The efficacy of recombinant factor VIIa (rFVIIa) as a bypassing agent in patients with haemophilia has been demonstrated in case studies and clinical trials. However, the perception of a short plasma half-life and consequent need for repeated daily injections means that long-term prophylaxis could potentially be limiting. Canine haemophilia models using a gene transfer approach have been used to evaluate the continuous expression of FVIIa in dogs. These studies show improvement in measurable bleeding parameters that have important clinical ramifications for patients with haemophilia. The combination of gene transfer as the method of delivery and FVII as the transgene overcomes issues associated with the short plasma half-life of rFVIIa, and represents a potentially attractive novel approach to haemostasis in patients with haemophilia and other platelet disorders.

\section{Keywords}

animal model; FVIIa; gene transfer; haemophilia; prophylaxis

\section{Introduction}

Antibodies (inhibitors) that inhibit or inactivate replacement therapy with factor VIII (FVIII) (or factor IX [FIX]) remain one of the most serious complications affecting patients with haemophilia [1-3]. The development of inhibitors occurs in 20-30\% of patients with haemophilia A and in around 5\% of patients with haemophilia B [4]. In those patients who have high-responding inhibitors, traditional factor replacement therapy is often ineffective [5] and patients are treated with bypassing therapy [6].

Recombinant factor VIIa ([rFVIIa]; NovoSeven ${ }^{\circledR}$; NovoNordisk, Bagsvaerd, Denmark) is considered a bypassing agent [5,7] meaning that it bypasses the usual coagulation process or pathways that utilize FVIII and FIX to generate a blood clot. Data from a series of case

(C) 2010 Blackwell Publishing Ltd

Correspondence: Achim Obergfell, NovoNordisk, Andreasstrasse 15, Zurich Oerlikon, CH-8050, Switzerland. Tel.: +4143 22223 43; fax: +4143 22243 43; aobe@ novonordisk.com.

Disclosures

Achim Obergfell and Mirella Ezban are employees of Novo Nordisk. Timothy Nichols has received funds for research from Novo Nordisk. 
reports in secondary prophylaxis show that rFVIIa in haemophilia patients with inhibitors and a high bleeding tendency reduces the number and severity of bleeds and improves joint status/quality of life, without evidence of pathological thrombosis or adverse events [8-12]. A further study (the only randomized, prospective clinical trial of a bypassing agent) has investigated the efficacy and safety of rFVIIa for secondary prophylaxis of 22 patients with severe haemophilia A or B with inhibitors. In this study, bleeding frequency was reduced during prophylaxis with $90 \mu \mathrm{g} \mathrm{kg}^{-1}$ or $270 \mu \mathrm{g} \mathrm{kg}^{-1}$ daily and the majority of the reduction in bleeds was maintained during post-prophylaxis, with no thromboembolic events reported [13]. Additionally, rFVIIa has been used effectively to control bleeding in haemophilia patients with inhibitors undergoing surgery [14].

Irrespective of these data outlining the efficacy and safety of rFVIIa in prophylactic regimens, the perception remains that the short plasma half-life [15] and consequent need for repeated daily injections will limit its use for prolonged periods of prophylaxis [16]. This article reviews the work of several investigators who have used animal models of genebased therapy approaches to study the effect of continuous expression of FVIIa [17].

\section{Murine models of FVII transgene therapy}

Relatively few data on the haemostatic effects of continuously elevated FVIIa levels are available. To address this issue, Margaritis et al. tested a modified FVIIa transgene in a murine model to investigate whether FVIIa continuously expressed is able to achieve adequate haemostasis without promoting pathological thrombosis [17]. In this study a human FVII (hFVII) transgene containing a cleavage site for the intracellular protease, paired amino acid-cleaving enzyme/furin, was shown to be correctly processed intracellularly and secreted as human-activated FVII (hFVIIa). This construct was introduced into a recombinant adeno-associated virus (AAV) vector and shown to direct long-term, high-level expression in normal mice. Levels of approximately $2 \mu \mathrm{g} \mathrm{mL} \mathrm{L}^{-1}$ could be obtained; these levels are comparable to those obtained in patients after dosing with rFVIIa [15].

Using these data as a guide, the hFVII was switched to a vector encoding an engineered murine FVII (mFVII) to resolve an important difference between the two species of insufficient binding of hFVIIa to murine tissue factor. The $\mathrm{mFVII}$ vector was introduced into the hepatic circulation of normal and haemophilia B C57BL/6 animals resulting in longterm expression of mFVIIa. A sustained reduction in both the prothrombin clotting time (PT) and activated partial thromboplastin time (aPTT) was observed. The clotting times were lowered to levels typically observed in the normal range. The reduction in aPTT is because of the high concentration of circulating rFVIIa which, through interaction with phospholipids in the aPTT reagents, is capable of inducing thrombin generation and results in a shortening of the clotting time. In addition, the use of a murine transgene allowed the evaluation of haemostasis in the haemophilic mouse model in response to a haemostatic challenge in vivo (tail clip assay). The tail clip assay showed that the mFVIIa levels achieved (approximately $1 \mu \mathrm{g} \mathrm{mL}^{-1}$ ) were sufficient to reduce bleeding substantially in all treated haemophilia B mice compared with untreated animals. No influence on survival (at 6 months) was observed, and no biochemical evidence of increased risk of thrombosis compared with untreated haemophilia B controls was reported [17].

The modified FVII transgene permits the evaluation of whether low levels of FVIIa, continuously expressed, would be sufficient to achieve haemostasis without promoting thrombosis - a hypothesis tested in a study by Aljamali et al. [18]. In this study transgenic mice that expressed a range of mFVIIa levels (categorized as low and high expressors based on plasma levels of mFVIIa) were generated and crossed with mice with haemophilia $\mathrm{B}$. The 
data showed that when mFVIIa was continuously expressed in transgenic mice, even low levels were sufficient to improve haemostasis and expression of mFVIIa at levels in the range of $0.3 \mu \mathrm{g} \mathrm{mL}^{-1}(6 \mathrm{nM})$ to $1.5 \mu \mathrm{g} \mathrm{mL}^{-1}(30 \mathrm{nM})$ were compatible with a normal lifespan. However, over-expression of mFVIIa at levels exceeding $2 \mu \mathrm{g} \mathrm{mL}^{-1}(40 \mathrm{nM})$ caused early mortality in normal and haemophilic mice possibly because of cardiac and pulmonary side effects. These results suggested ranges for haemostatic efficacy of continuously expressed mFVIIa and defined upper limits for safety in mice [18].

\section{Canine model of gene-based FVIla expression}

To investigate whether the positive effects of gene transfer observed in murine models translate to large animals, Margaritis et al. [16] used the canine haemophilia model to evaluate gene-based, AAV-mediated continuous expression of canine FVIIa (cFVIIa) as a treatment for haemophilia. Canine models have been extensively applied in haemostasis research and are considered valid models in relation to human coagulation factor deficiencies, particularly haemophilia A and B.

\section{Efficacy of AAV-mediated cFVII gene transfer}

A serotype $8 \mathrm{AAV}$ vector (AAV8-cFVII) was generated that directed expression of cFVIIa via a liver-specific promoter in a similar manner to that described in previous murine studies $[17,18]$. To test the AAV vector, tail vein vector administration in mice with haemophilia was performed, which demonstrated that expression of cFVIIa resulted in long-term, sustained haemostatic functionality as shown by normalization of the haemophilic aPTT and concurrent supraphysiological shortening in the PT. The next experiment was the infusion of the AAV8-cFVII vector (6.25-12.5E13 $\left.\mathrm{vg} \mathrm{kg}^{-1}\right)$ into haemophilia A and haemophilia B dogs which led to a long-term, stable reduction for both whole blood clotting time (WBCT) and PT as a result of sustained canine FVIIa (cFVIIa) expression that ranged between 1.3 and $2.6 \mu \mathrm{g} \mathrm{mL}^{-1}$ ( $P<0.03$ vs. normal or untreated haemophilia A or B dogs). Moreover, thromboelastography analysis of whole blood from these dogs with haemophilia A or B showed improved clot dynamics and a sustained, near-normalization of the reaction time (time to initial fibrin formation) vs. normal dogs [16].

\section{Spontaneous bleeding episodes in AAV-cFVII-treated haemophilic dogs}

The number of spontaneous bleeds was also evaluated as a measure of clinical efficacy as dogs with haemophilia are known to exhibit these types of bleeds several times per year. Untreated dogs with haemophilia A and B observed concurrently within the study exhibited 12 and 21 spontaneous bleeds in 36 and 60 months of observation respectively. In comparison, during 34-month (haemophilia B) and 45-month (haemophilia A) observation periods, the AAV8-cFVII-treated dogs did not exhibit any spontaneous bleeding episodes [16].

\section{Safety of long-term cFVIla expression in AAV-treated haemophilic dogs}

Analysis showed that serum chemistries for liver and kidney functions were within the normal range in all dogs before and after gene transfer. Platelet counts were within the normal range throughout the study and levels of thrombin-antithrombin (TAT) complex and D-dimer levels (elevated levels of which are indicators of intravascular fibrin forming processes) did not change significantly throughout the observation period relative to preAAV administration levels. Fibrinogen levels displayed some variability. These findings in a limited numbers of dogs indicate that continuous cFVIIa expression had a good safety profile in this study [16]. 


\section{Discussion and conclusion}

Continuous expression of cFVIIa in dogs with haemophilia provides proof-of-concept for the gene transfer approach investigated. Data reported are similar to those observed in transgenic haemophilic mice (mFVIIa) and haemophilic mice receiving AAV-mFVIImediated gene transfer $[17,18]$. Circulating plasma levels of cFVIIa in the range of 1.5-2.5 $\mu \mathrm{g} \mathrm{mL}^{-1}$ were obtained, and led to supraphysiologically reduced PT as well as significantly improved throm-boelastography measurements. More importantly, no spontaneous bleed was observed in these dogs. Taken together these results, in conjunction with the murine model data, suggest haemostatic efficacy similar to levels achieved with bolus rFVIIa infusion in humans [16].

Animal model data demonstrate that liver-directed, AAV8-mediated gene transfer of mFVII or cFVII in haemophilia A and B leading to continuous expression of FVIIa is effective and safe in the short-and medium-term, and results in improvement in measurable bleeding parameters that have important clinical ramifications for patients with haemophilia. The combination of gene transfer as the method of delivery and FVII as the transgene obviates any issues relating to tolerance (immunological) and the short plasma half-life of rFVIIa, and represents a potentially attractive novel approach to haemostasis in patients with haemophilia and other platelet disorders.

\section{References}

1. Saint-Remy JM, Lacroix-Desmazes S, Oldenburg J. Inhibitors in haemophilia: pathophysiology. Haemophilia. 2004; 10(Suppl 4):146-51. [PubMed: 15479388]

2. Haya S, Moret A, Cid AR, et al. Inhibitors in haemophilia A: current management and open issues. Haemophilia. 2007; 13(Suppl 5):52-60. [PubMed: 18078398]

3. Jiménez-Yuste V, Alvarez MT, Martín-Salces M, et al. Prophylaxis in 10 patients with severe haemophilia A and inhibitor: different approaches for different clinical situations. Haemophilia. 2009; 15:203-9. [PubMed: 19149850]

4. Berntorp E, Shapiro A, Astermark J, et al. Inhibitor treatment in haemophilias A and B: summary statement for the 2006 international consensus conference. Haemophilia. 2006; 12(Suppl 6):1-7. [PubMed: 17123387]

5. DiMichele DM, Hoots WK, Pipe SW, Rivard GE, Santagostino E. International workshop on immune tolerance induction: consensus recommendations. Haemophilia. 2007; 13(Suppl 1):1-22. [PubMed: 17593277]

6. Leissinger CA. Prevention of bleeds in hemophilia patients with inhibitors: emerging data and clinical direction. Am J Hematol. 2004; 77:187-93. [PubMed: 15389908]

7. Hedner U. Recombinant factor VIIa: its background, development and clinical use. Curr Opin Hematol. 2007; 14:225-9. [PubMed: 17414211]

8. Young G, McDaniel M, Nugent DJ. Prophylactic recombinant factor VIIa in haemophilia patients with inhibitors. Haemophilia. 2005; 11:203-7. [PubMed: 15876264]

9. Saxon BR, Shanks D, Jory CB, Williams V. Effective prophylaxis with daily recombinant factor VIIa (rFVIIa-Novoseven) in a child with high titre inhibitors and a target joint. Thromb Haemost. 2001; 86:1126-7. [PubMed: 11686341]

10. Cooper HA, Jones CP, Campion E, Roberts HR, Hedner U. Rationale for the use of high dose rFVIIa in a high-titre inhibitor patient with haemophilia B during major orthopaedic procedures. Haemophilia. 2001; 7:517-22. [PubMed: 11554944]

11. Auerswald G. Long-term prophylaxis in congenital haemophilia with inhibitors - experiences with rFVIIa. Hamostaseologie. 2007; 27:123-8. [PubMed: 17479176]

12. Blatny J, Kohlerova S, Zapletal O, Fiamoli V, Penka M, Smith O. Prophylaxis with recombinant factor VIIa for the management of bleeding episodes during immune tolerance treatment in a boy with severe haemophilia A and high-response inhibitors. Haemophilia. 2008; 14:1140-2.

[PubMed: 18540894] 
13. Konkle BA, Ebbesen LS, Erhardtsen E, et al. Randomized, prospective clinical trial of recombinant factor VIIa for secondary prophylaxis in hemophilia patients with inhibitors. J Thromb Haemost. 2007; 5:1904-13. [PubMed: 17723130]

14. Shapiro AD, Gilchrist GS, Hoots WK, Cooper HA, Gastineau DA. Prospective, randomised trial of two doses of rFVIIa (NovoSeven) in haemophilia patients with inhibitors undergoing surgery. Thromb Haemost. 1998; 80:773-8. [PubMed: 9843170]

15. Hedner U. Recombinant factor VIIa (Novoseven) as a hemostatic agent. Semin Hematol. 2001; 38:43-7. [PubMed: 11735110]

16. Margaritis P, Roy E, Aljamali MN, et al. Successful treatment of canine hemophilia by continuous expression of canine FVIIa. Blood. 2009; 113:3682-9. [PubMed: 19109232]

17. Margaritis P, Arruda VR, Aljamali M, et al. Novel therapeutic approach for hemophilia using gene delivery of an engineered secreted activated Factor VII. J Clin Invest. 2004; 113:1025-31. [PubMed: 15057309]

18. Aljamali MN, Margaritis P, Schlachterman A, et al. Long-term expression of murine activated factor VII is safe, but elevated levels cause premature mortality. J Clin Invest. 2008; 118:1825-34. [PubMed: 18398505] 